\title{
Explanation of black hole radiation by applying the Lense-Thirring Effect and extending the Bardeen-Petterson Effect
}

Written in Keighley

The $7^{\text {th }}$ of February 2018

By Professor Alexandre GEORGES

Professor Alexandre GEORGES

51 Redcliffe Street

Keighley

England

United Kingdom 


\section{Summary}

Summary Page 2

Preamble Page 3

Part I - Lense-Thirring Effect and Bardeen-Petterson Effect Page 4

Subpart 1 - Lense-Thirring Effect Page 6

Subpart 2 - Bardeen-Petterson Effect Page 7

Part II - Interpretation through Theory X Page 8

Subpart 1 - Theoretical bases needed Page 10

Subpart 2 - Application of the Atropic Effect Page 11

Subpart 3 - Graphical representation Page 13

Part III - Possible verification of the Atropic Effect Page 14

Subpart 1 - Proposal of method Page 16

Subpart 2 - Possible biases and minimum expected observations Page 17

Conclusions Page 18

Brief references Page 19 


\section{Preamble}

The purpose of this publication is to demonstrate that there may be a relativistic explanation for Hawking radiation. Presented this way, it does not look exceptional, but it would require that a quantity of energy in the form of light would correspond to a specific deformation of the Space-Time. The explanation in question consists of an extension of the Bardeen-Petterson Effect (BPE), a fairly simple and verifiable application of the LenseThirring Effect (LTE). We will call this phenomenon, at the origin of the generation of black hole radiation, the Atropic Effect (AE), named after one of the moirs of Greek mythology: Atropos ("the inevitable"). 
Part I - Lense-Thirring Effect and Bardeen-Petterson Effect 


\section{Bureau du Professeur GEORGES}

According to Newtonian mechanics, the gravitation exerted by a body propagates instantly and depends only on the distance between the bodies that influence each other and their respective masses, this being consistent with the principle that two bodies in motion "perceive" the space in the same way. The effect of gravitation exerted by a body then spreads instantly to all space and is not influenced by its movement but by its distance from other bodies. 
Subpart 1 - Lense-Thirring Effect

In General Relativity, a gravity effect - tested and measured by Gravity Probe B satellite - is the Lense-Thirring Effect, explaining that, around a massive rotating body, space and time are somehow "dragged" with the rotation movement.

Thus, when a body is in rotation on itself, in addition to the gravitational effect that modifies the space-time, its rotation modifies a little the geometry of the gravitational field that it emits and which propagates at the speed of light. 


\section{Bureau du Professeur GEORGES}

Subpart 2 - Bardeen-Petterson Effect

The Bardeen-Petterson Effect, around a fast-spinning compact object, deforms an accretion disk inclined in the equatorial plane of the rotating body. The viscous forces cause the accretion to divide into two distinct regions: an internally aligned accretion disk and an external inclined accretion disk. The transition between the two occurs at a characteristic radius which depends on the mass and the angular momentum of the central object and possibly on the rate of accretion through the disc. ${ }^{1}$

A gaseous accretion disk that is tilted with respect to a spinning black hole will experience Lense-Thirring precession. Because the precession rate varies with distance from the black hole, the disk will wrap up, until viscosity forces the gas into a new plane, aligned with the black hole's spin axis.

It might be interesting to see, applying principles from Theory $\mathrm{X}$, if these effects can be extended to explain the radiation of black holes. 
Part II - Interpretation through Theory X 


\section{Bureau du Professeur GEORGES}

First of all, it is necessary to explain what a unifying theory of Physics is, in what it consists. To put it simply, it consists of a unification of the two main branches of Physics: General Relativity, theory of gravitation, describing the world of the infinitely large, and Quantum Mechanics, theory of electromagnetism and nuclear forces, describing the world of the infinitely small. Thus, a unifying theory of Physics would consist in the discovery of the fundamental force at the origin of the gravitational, electromagnetic, strong nuclear, and weak nuclear forces.

Then, it is necessary to remember that, contrary to the String Theory and the Loop Quantum Gravity, which try to explain by principles specific to the Quantum Mechanics the gravitational force, the Theory $\mathrm{X}$, it, is a relativistic theory, establishing the following laws:

I - The gravitational force is the fundamental force and is exerted between quantities of energy, like the photon, particle with a quantity of energy and deprived of mass, deforming in a specific way the geometry of Space-Time.

II - The entirety of the particles of matter of the Universe consists of quantities of energy.

III - There is no form of randomness in the Universe.

If the Theory $\mathrm{X}$ is correct, it must in particular be able to explain the origin of the Hawking radiation and explain how they are generated by the gravitational singularities. ${ }^{2}$ 


\section{Bureau du Professeur GEORGES}

Subpart 1 - Theoretical bases needed

According to Theory $\mathrm{X}$, a photon corresponds to a deformation of the geometry of Space-Time.

$$
g_{i j}=-\left(1-\frac{2 G M}{c^{2} r}\right) c^{2} d t^{2}+\left(1-\frac{2 G M}{c^{2} r}\right)^{-1} d r^{2}+r^{2}\left(d \Theta^{2}+\sin \Theta d \Phi^{2}\right)
$$

The metric describes the geometry of space-time, deformed by the gravitational field of a spherical and immobile mass object.

The problem occurs when we study the case of the photon. Since it has no mass, we project a flat Space-Time. Now, $\mathrm{E}=\mathrm{Mc}^{2}$, we can then replace $\mathrm{M}$, in the metric, by $\mathrm{E} / \mathrm{c}^{2}$. Let's see our new metric expression:

$$
\gamma_{i j}=-\left(1-\frac{2 G E}{c^{4} r}\right) c^{2} d t^{2}+\left(1-\frac{2 G E}{c^{4} r}\right)^{-1} d r^{2}+r^{2}\left(d \Theta^{2}+\sin ^{2} \Theta d \Phi^{2}\right)
$$

We are here faced with a particle that does not distort Space Time because of its mass, but because of its quantity of energy. This is a specific deformation of Space Time.

Also, the equations of Theory $\mathrm{X}$ recognize as possible the principle of body-curvature reciprocity, according to which a body would deform the geometry of Space-Time as much as an identical curvature would "produce" an identical body. ${ }^{2}$ 


\section{Bureau du Professeur GEORGES}

Subpart 2 - Application of the Atropic Effect

The application of the Atropic Effect consists of an application of LTE and BPE in the case of black holes.

As indicated in Part I:

Around a massive rotating body, space and time are somehow "dragged" with the rotation movement.

Thus, when a body is in rotation on itself, in addition to the gravitational effect that modifies the space-time, its rotation modifies a little the geometry of the gravitational field that it emits and which propagates at the speed of light.

The Bardeen-Petterson Effect, around a fast-spinning compact object, deforms an accretion disk inclined in the equatorial plane of the rotating body. The viscous forces cause the accretion to divide into two distinct regions: an internally aligned accretion disk and an external inclined accretion disk. The transition between the two occurs at a characteristic radius which depends on the mass and the angular momentum of the central object and possibly on the rate of accretion through the disc. ${ }^{1}$

A gaseous accretion disk that is tilted with respect to a spinning black hole will experience Lense-Thirring precession. Because the precession rate varies with distance from the black hole, the disk will wrap up, until viscosity forces the gas into a new plane, aligned with the black hole's spin axis.

In order to apply this to the singularities, it is necessary, on the one hand, to consider their rotation and, on the other hand, to consider the effect of the rotation on the curvature of the space-time and the consequence of this alteration of the deformation at the poles of the singularity, crossed by the axis of rotation of the black hole, inclined perpendicularly to the accretion disk.

Now, imagine that the internal structure of the black hole, which is on the other side of the event horizon, is not a compact object, but another vortex of quanta of energy - given their speed - whose light could not really escape, which we will call the heart. By applying LTE, the geometry of the Space-Time would be altered by the rotation of the singularity core and, according to the BPE, this "dragging" would result in a higher concentration of quanta on a 


\section{Bureau du Professeur GEORGES}

plan perpendicular to the axis of rotation and less important to the poles traversed by said axis of rotation. We will call this plan the critical accretion disk (CAD), given that such an accretion of quanta would have the consequence, according to Theory $\mathrm{X}$, of allowing the propagation of a gravitational field, in the same way as for matter, which would be, given the heterogeneity of the "gravitational bath", not a perfect plan.

Let's now translate this vortex of quanta of energy by a vortex of point spatial contractions, as indicated by Theory $\mathrm{X}$. The spatial contraction of the CAD would then be higher than that of the axis of rotation.

A quantum of energy then enters the heart by the CAD plan, it is attracted towards the center of the heart of the singularity and reaches it. At this moment, nothing prevents it to cross it, but the extreme contraction spatial the CAD would not allow him to leave the singularity if he does not return to the horizon of events by following a geodesic that would allow it, and therefore a path not curved by the greater attractiveness of the CAD.

The only way for a quantum of energy to leave the heart would be to follow a trajectory extremely close to the axis of rotation of the black hole, where the Space-Time "dragging" would be weak and where it would not "toggle" again to the CAD.

Considering the heart of a singularity as a vortex of point spatial contractions leads us to imagine a heart from which, at the poles traversed by the axis of rotation of the black hole, space contractions would escape.

According to Theory $\mathrm{X}$, the projection at the poles of spatial contractions would be translated by jets of quanta, radiation by the poles of the singularity, corresponding to its loss in mass. This generation of radiation by altering the curvature of space-time due to the rotation of the singularity would then be responsible for the evaporation of the black holes.

The same mathematical concepts as those used for the Lense-Thirring Effect would be applied to describe this phenomenon. 


\section{Bureau du Professeur GEORGES}

Subpart 3 - Graphical representation

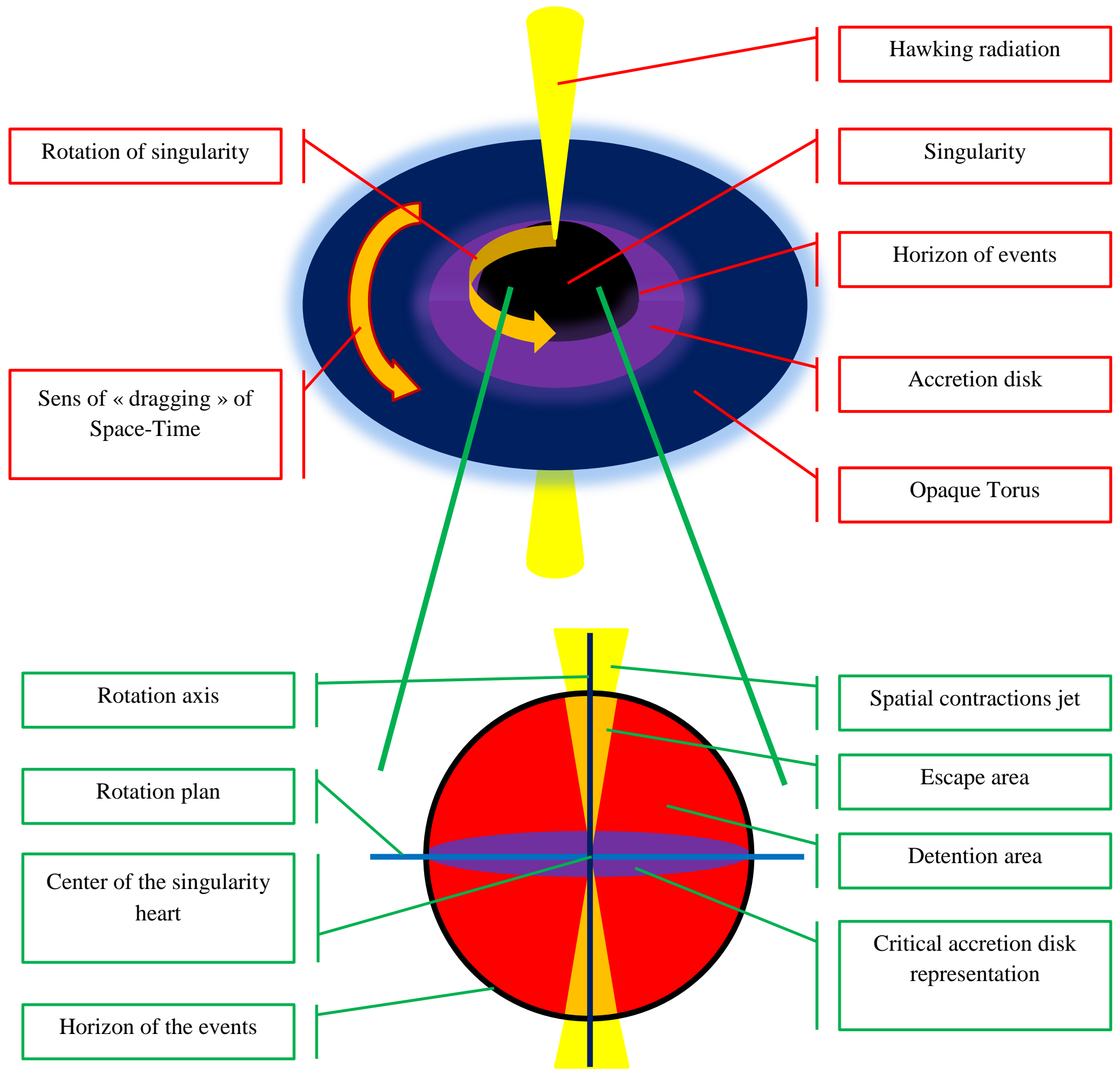


Part III - Possible verification of the Atropic Effect 


\section{Bureau du Professeur GEORGES}

In order to determine if the Atropic Effect is really at the origin of the radiation of the black holes and therefore of their inevitable evaporation, we first need if the predictions of radiation emission from the model that is the Theory $\mathrm{X}$ correspond to the actual emissions of black holes. 


\section{Bureau du Professeur GEORGES}

Subpart 1 - Proposal of method

A series of observations could be conducted on some specimens of each variety of black holes.

We would then analyze, for each specimen, the alteration of the deformation of the geometry of Space-Time around the black hole, in order to determine, taking into account the mass of the singularity and its disks, the speed of rotation of said black hole. This would make it possible to establish the importance of the jets of space contractions emanating theoretically from its poles and to establish the amount of energy that would correspond to them. We could finally compare the predictions with the results of the observation of the emitted radiation. If the predictions were correct, then the Atropic Effect would be the expected explanation of the emission of Hawking radiation.

It would be a high precision job, but it would be workable. 


\section{Bureau du Professeur GEORGES}

Subpart 2 - Possible biases and minimum expected observations

Given the great precision required to arrive at a perfect result and the importance of the factors that can intervene during the experiment, the observations must above all highlight the correlations between the different predictions and their respective results, in order to avoid any bias. The correspondence between the predictions and the results could lead, once all bias has been removed, to demonstrate whether the application of LTE and BPE is viable in the case of a black hole, and therefore if the Atropic Effect is at origin of the radiation of black holes and their evaporation. 


\section{Conclusions}

If the predictions correspond to the results obtained by the observation, it would mean that the Atropic Effect is indeed at the origin of the radiation of the black holes and their evaporation and would have as consequence to validate the cosmological model that is Theory $\mathrm{X}$. It would then be the unifying theory of physics.

\section{Professor Alexandre GEORGES}

Theoretical Physicist 


\section{Brief references}

The following statements are not there to justify the quality of the information used and provided, but to respect the work of the researchers who produced what has served to write this article here and refer to it, in case its readers would need more details regarding this work. There will therefore be no mention here of commonly accepted and well-known models and facts, such as General Relativity or the Lense-Thirring Effect.

${ }^{1}$ BARDEEN-PETTERSON EFFECT AND QUASI-PERIODIC OSCILLATIONS IN XRAY BINARIES (2000) by P. CHRIS FRAGILE, GRANT J. MATHEWS, AND JAMES R. WILSON

${ }^{2}$ Publication relative à la Théorie X Réédition partielle (2017) by A. GEORGES 